Virginia Commonwealth University

VCU Scholars Compass

1993

\title{
Analysis of homogeneous nucleation data of polar molecules: Vapor association, dipole orientation, and elongated clusters
}

D. Wright

Virginia Commonwealth University

M.S. El-Shall

Virginia Commonwealth University, mselshal@vcu.edu

Follow this and additional works at: http://scholarscompass.vcu.edu/chem_pubs

Part of the Chemistry Commons

Wright, D., and El-Shall, M. S. Analysis of homogeneous nucleation data of polar molecules: Vapor association, dipole orientation, and elongated clusters. The Journal of Chemical Physics, 98, 3369 (1993). Copyright (c) 1993 American Institute of Physics.

\section{Downloaded from}

http://scholarscompass.vcu.edu/chem_pubs/50

This Article is brought to you for free and open access by the Dept. of Chemistry at VCU Scholars Compass. It has been accepted for inclusion in Chemistry Publications by an authorized administrator of VCU Scholars Compass. For more information, please contact libcompass@vcu.edu. 


\title{
Analysis of homogeneous nucleation data of polar molecules: Vapor association, dipole orientation, and elongated clusters
}

\author{
D. Wright and M. S. El-Shall ${ }^{\text {a) }}$ \\ Department of Chemistry, Virginia Commonwealth University, Richmond, Virginia 23284-2006
}

(Received 21 August 1992; accepted 26 October 1992)

\begin{abstract}
In this paper we present the results of several theoretical models examined in order to explain the observed systematic deviations of the homogeneous nucleation of highly polar substances from the predictions of the classical nucleation theory $(\mathrm{CNT})$. It is found that molecular association in acetronitrile or nitromethane vapor cannot explain the observed higher supersaturations. Similarly, the use of virial coefficient data to obtain a size dependent surface energy correction factor does not reproduce the experimental results. It is shown that orientation of the surface dipoles within the spherical droplets results in higher supersaturations in agreement with the experimental findings. We also generalize the CNT for elongated clusters and show that these clusters can allow for favorable end-toend and antiparallel alignments of the dipoles. It is found that for reasonable agreement with experiment, acetonitrile, and benzonitrile clusters require a larger degree of elongation compared to nitromethane and nitrobenzene clusters. These results are discussed in relation to molecular simulations of bulk liquids and clusters.
\end{abstract}

\section{INTRODUCTION}

In a recent letter, Wright et al. ${ }^{1}$ reported that the homogeneous nucleation of acetronitrile does not follow the predictions of the classical nucleation theory (CNT). The experimental measurements of the critical supersaturation over a wide range of temperature yielded values $40 \%-50 \%$ higher than the calculated supersaturations from the CNT. This study has been extended to include other polar molecules such as cyanobenzene, nitromethane, and nitrobenzene, and similar behavior has been observed. ${ }^{2,3}$ The results indicate that the nucleation rates of highly polar compounds are slower by several orders of magnitude than the predictions of the CNT. As the CNT has been a fairly good predictor of critical supersaturations for the onset of nucleation for a wide variety of compounds we have examined several possible proposals to explain the observed deviations for highly polar molecules. The purpose of this paper is to present the results of the models examined and compare them with those obtained within the scope of the CNT.

The CNT assumes the vapor to be composed only of individual molecules and neglects any effects arising from the presence of small, relatively stable complexes. It has been observed, ${ }^{4-6}$ however, that these effects can be significant in highly associated vapors such as the smaller carboxylic acids where the experimental supersaturations lie well above the predictions of the CNT. Katz, Saltzburg, and Reiss (KSR) (Ref. 7) have presented a more general form of the CNT which incorporates the effects of vapor association. A key prediction of the KSR theory is that the overall effect of association is to decrease the rate of nucleation and therefore to increase the critical supersaturation. This model has been successfully applied to carboxylic acids and reasonable agreement with experiment has been

${ }^{2}$ To whom correspondence should be addressed. found. If the polar compounds of the present study were sufficiently associated in the supersaturated vapor, this could possibly explain the observed deviations from the CNT.

Dillmann and Meier have recently developed a "refined droplet approach" to the homogeneous nucleation problem. ${ }^{8,9} \mathrm{~A}$ significant feature of this model is the use of virial coefficient data to obtain a size dependent correction to the bulk surface tension for the embryonic droplets. This approach has been quite successful for alcohols, water, and nonane and gives more accurate predictions than the CNT. Since the second virial coefficient reflects the dipole-dipole interaction between isolated pairs of molecules, the application of this model to clusters of highly polar molecules may capture this feature of the intermolecular forces which is not explicitly contained within the CNT.

The recognition that the CNT does not take proper account of the dipole-dipole interaction in calculating the free energy of the clusters raises interest in the dipole orientation model. According to this proposal, polar molecules may exhibit a preferential orientation at the droplet surface leading to a surface tension that differs from the bulk surface tension assumed by the CNT. By explicitly considering the effects of dipole-dipole interaction on the surface free energy of the embryos, Abraham ${ }^{10}$ has derived an expression for a size dependent surface tension for clusters with oriented surface dipoles. The predictions of this model depend upon the size of the dipole moment, the size of the cluster, and the separation between surface molecules in the cluster.

One other possibility that follows as a consequence of dipole orientations is that for highly polar molecules the embryonic droplets may become somewhat elongated due to energetically favorable head-to-tail and antiparallel alignments of the dipoles. This would tend to give the clusters a prolate spheriodal shape resulting in an increase in the surface-to-volume ratio and thus an increase in surface 
free energy. The CNT can be generalized so that the assumption of spherical embryos is relaxed and the clusters can take on elongated shapes.

The outline of the paper is as follows: first, we apply the KSR model to determine the effect of vapor association on the homogeneous nucleation of acetonitrile and nitromethane. In Sec. III, we examine the application of the refined droplet model of Dillmann and Meier to acetonitrile and nitromethane since virial coefficient data are only available for these vapors. Section IV contains the oriented dipoles treatment for the studied compounds namely, acetonitrile, nitromethane, benzonitrile, and nitrobenzene. Finally, in Sec. V we derive the rate equation of the CNT for nonspherical clusters and apply the resulting equation to the highly polar molecules.

\section{KSR ASSOCIATION}

Katz, Saltzburg, and Reiss ${ }^{7}$ have presented a more general form of the CNT which incorporates the effects of vapor association. The KSR equation for the rate of nucleation in an associated vapor is given by

$$
\begin{aligned}
J= & (q / d)\left(2 N_{A}^{3} \sigma M / \pi\right)^{1 / 2}\left(S P_{e} / R T\right)^{2} \sum_{i=1}^{m} i^{3 / 2} x_{i} \\
& \times \exp \left\{-16 \pi N_{A} M^{2} \sigma^{3} /\left[3\left(d \ln S_{1}\right)^{2}(R T)^{3}\right]\right\},
\end{aligned}
$$

where clusters up to a size $m$ are included, where $m$ is the largest stable cluster present in the vapor. In Eq. (1), $J$ is the rate of nucleation (drops $\mathrm{cm}^{-3} \mathrm{~s}^{-1}$ ), $i$ is the number of molecules in an association cluster of size $i, x_{i}$ is the ratio $P_{i} / P$ (where $P_{i}$ is the partial pressure of the $i$-sized clusters and $P$ is the partial pressure of the vapor), $S$ is the supersaturation ratio $P / P_{e}$ (where $P_{e}$ is the equilibrium vapor pressure), and $S_{1}$ is the monomer supersaturation ratio $P_{1} / P_{1 e}$ (where $P_{1 e}$ is the equilibrium vapor pressure of the monomer only). Further, $q$ is the condensation coefficient, $d$ is the liquid density, $N_{A}$ is Avogadro's number, $\sigma$ is the bulk liquid surface tension, $M$ is the molecular weight, $R$ is the gas constant, and $T$ is the temperature. The preexponential factor differs slightly from the usual CNT expression due to the fact that an embryo may now gain or lose molecules in clusters of two or more, but the far more significant effect is that the cluster size distribution is altered by the presence of dimers, trimers, etc. This latter effect results in the monomer supersaturation being less than the total supersaturation and it is now $S_{1}$ that appears in the exponential. Thus the energy barrier is increased and higher supersaturations are required to induce nucleation. This theory has been successful in predicting the critical supersaturations for the smaller carboxylic acids. ${ }^{4-6}$ In order to check the accuracy of our KSR calculations, the temperature dependence of $S$ has been calculated for formic, acetic, and propanoic acids and similar results to those reported in Refs. 4-6 have been obtained.

The literature values of the dimerization constants for acetonitrile and nitromethane are included in Table I. Using these fits, the KSR predictions are calculated for $\mathrm{CH}_{3} \mathrm{CN}$ and $\mathrm{CH}_{3} \mathrm{NO}_{2}$ and the results are shown in Figs. 1 and 2, respectively. As the dimerization constants are rel-
TABLE I. The dimerization equilibrium constant $K_{2}$ (Torr $^{-1}$ ) and the second virial coefficient $B\left(\mathrm{~cm}^{3} / \mathrm{mol}\right)$ for $\mathrm{CH}_{3} \mathrm{CN}$ and $\mathrm{CH}_{3} \mathrm{NO}_{2} ; T_{r}=T /$ $T_{c^{*}}$

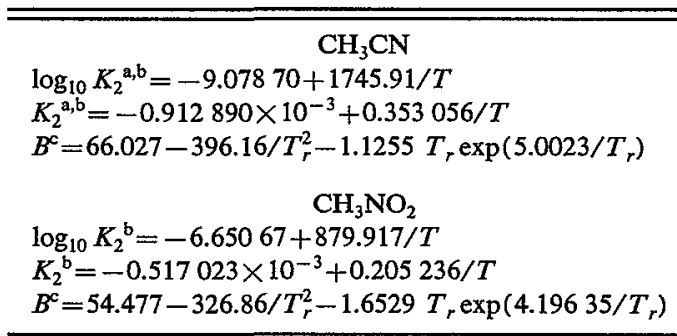

${ }^{a}$ Reference 20.

${ }^{\mathrm{b}}$ Reference 21 .

${ }^{\mathrm{c}}$ Reference 11.

atively small and only a few percent of the molecules are expected to exist as dimers, the KSR curves do not lie far above the predictions of the CNT which are well below the experimental results. The temperature dependence of these constants is typically expressed in the form $\log K$ vs $1 / T$, making extrapolation to lower temperatures less reliable when only a few points determine the fit. The significant increase in the predicted $S_{c}$ for $\mathrm{CH}_{3} \mathrm{CN}$ at lower temperatures is probably due to the extrapolation of the dimerization constant to temperatures almost $100 \mathrm{~K}$ below the lowest available literature value. When the data for the dimerization constant is fit to the form $K$ vs $1 / T$ (a slightly better fit) the curve for $\mathrm{CH}_{3} \mathrm{CN}$ is as close to the CNT as the one for $\mathrm{CH}_{3} \mathrm{NO}_{2}$ throughout the temperature range of the calculations. The KSR predictions for $\mathrm{CH}_{3} \mathrm{CN}$ for both of these fits are shown in Fig. 1.

The results in Figs. 1 and 2 indicate that the observed increase in the critical supersaturations of the polar molecules cannot be explained in terms of the KSR model for associating vapors. This is probably not a surprising result since success of the KSR equation is only expected if the vapor contains a large percentage of stable dimers and or higher clusters such as acetic acid vapor where at $T=386$ $\mathrm{K} \sim 56 \mathrm{~mol} \%$ dimer is present at saturation. However, with water and simple alcohols, the effect of association calculated by the KSR equation is very small owing to the small concentration of associated species in these vapors.

\section{THE REFINED DROPLET MODEL OF DILLMANN AND MEIER (DM)}

Using a semiphenomenological droplet model, the DM treatment ${ }^{8,9}$ takes the Gibbs free energy of formation of an embryo containing $i$ molecules to be given by

$$
\Delta G_{i}=\kappa_{i} \sigma s_{1} i^{2 / 3}+\tau k_{B} T \ln i-k_{B} T \ln \left(q_{0} V\right)-i k_{B} T \ln S,
$$

where $k_{B}$ is Boltzmann's constant, $s_{1}$ is the surface area of a spherical cluster containing $i$ molecules and where $\kappa_{i}, \tau$, and $q_{0}$ are model parameters. $\kappa_{i}$ is a size dependent correction to the surface tension and $\tau$ and $q_{0}$ are taken as arising from translational, rotational, vibrational, and configurational contributions to the droplet free energy. Using the 


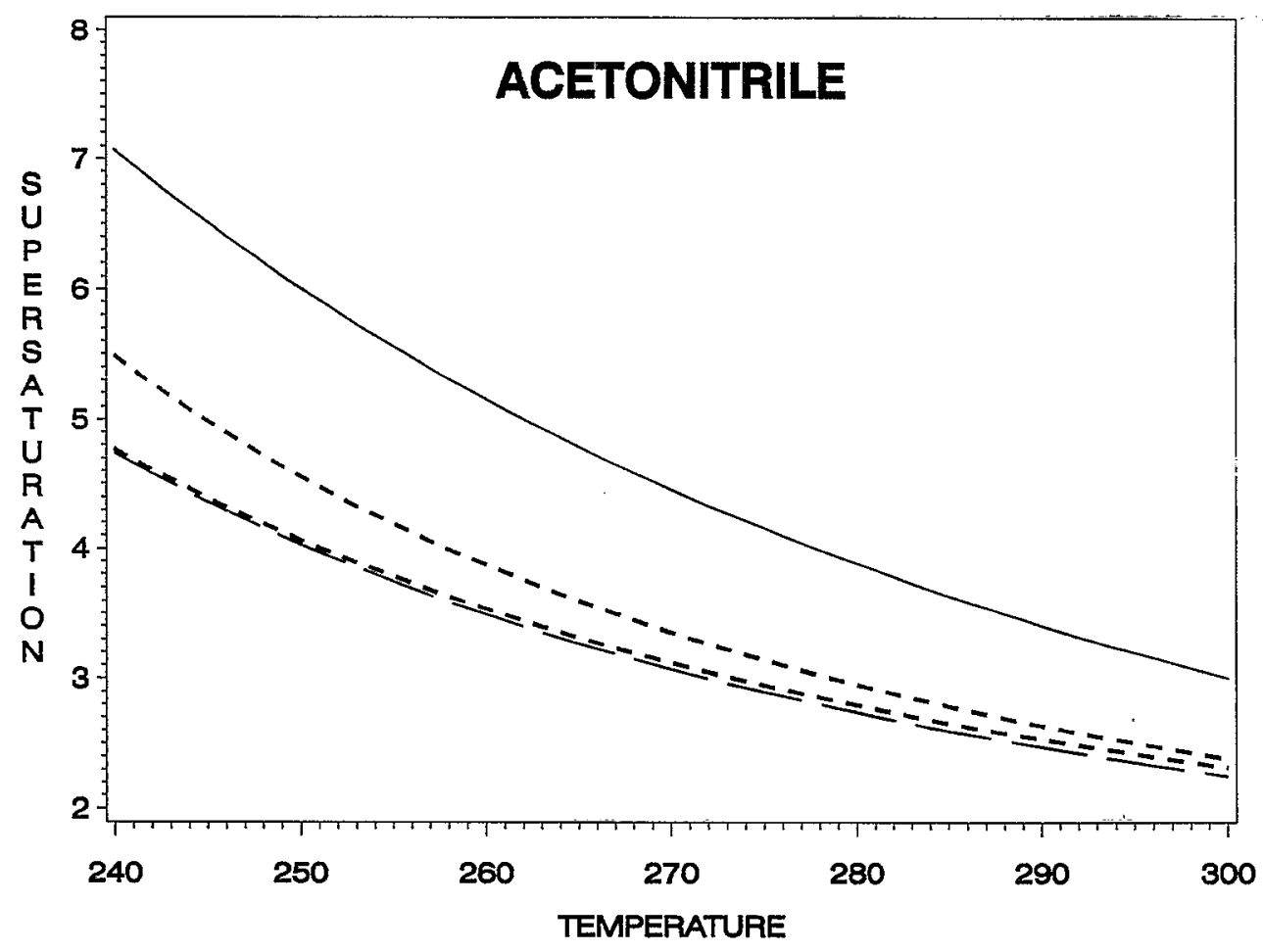

FIG. 1. Critical supersaturation vs temperature for acetonitrile. Solid line represents the experimental results, dashed line the CNT, and the dotted lines the predictions of the KSR model. (Upper and lower dotted lines correspond to the first and second fits listed in Table I, respectively.)

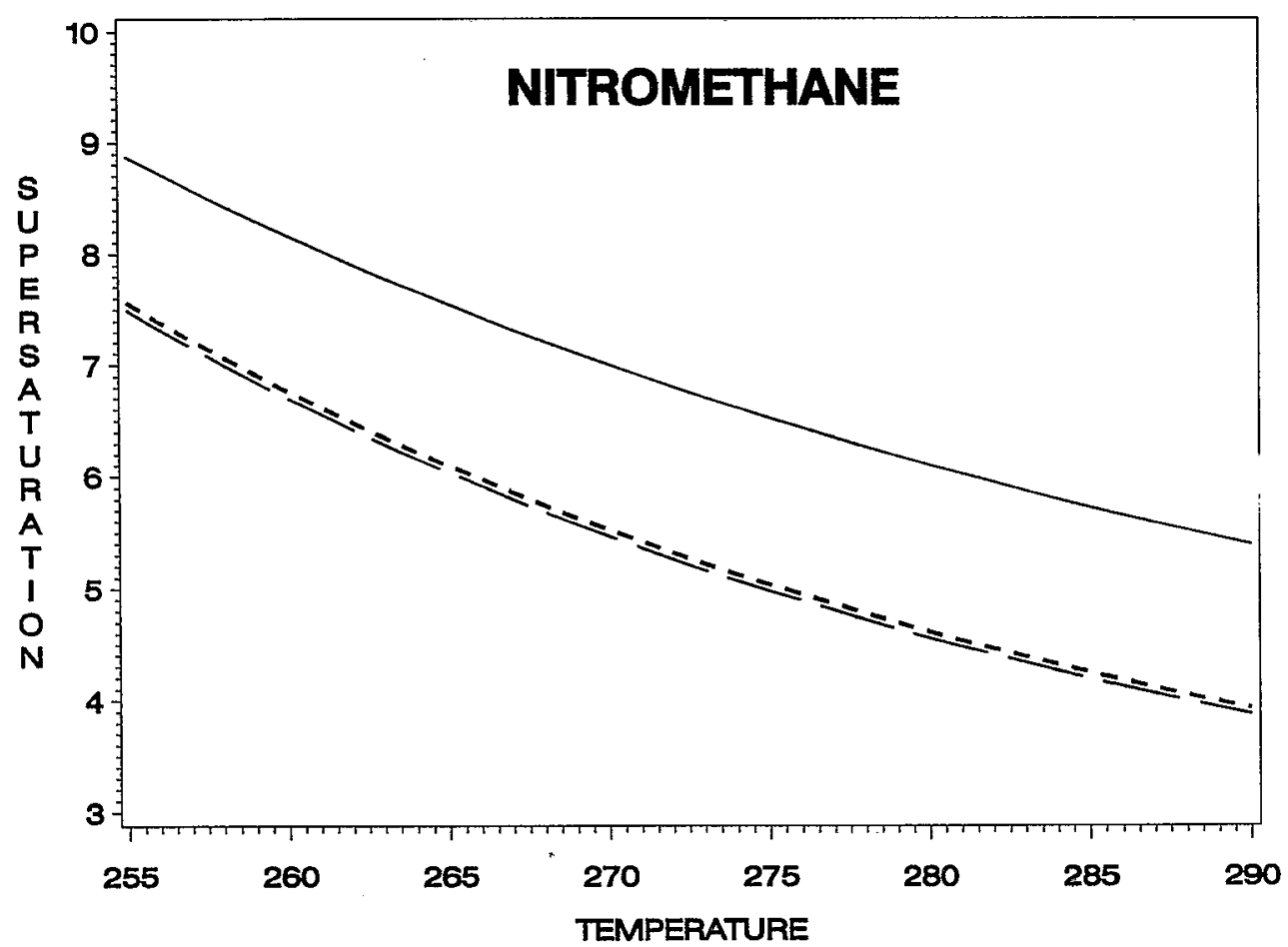

FIG. 2. Critical supersaturation vs temperature for nitromethane. Solid line represents the experimental results, dashed line the CNT, and the dotted line the predictions of the KSR model. (The two fits listed in Table I result in nearly identical lines.) 
following expression for the equilibrium concentration $\rho_{i, \text { eq }}$ of clusters containing $i$ molecules

$$
\rho_{i, \mathrm{eq}}=(1 / V) \exp \left[-\Delta G_{i} / k_{B} T\right],
$$

where $V$ is the system volume, along with Eq. (2), DM construct a thermal equation of state for the vapor which is given by

$$
\rho=q_{0} \sum_{i=1}^{\infty} \exp \left[-\kappa_{i} \theta i^{2 / 3}-(\tau-1) \ln i+i \ln S\right]
$$

where $\rho$ is the number density and $\theta$ is an abbreviation for $\sigma s_{1} / k_{B} T$. The dependence of $\kappa_{i}$ on cluster radius is taken to be of the form

$$
\kappa_{i}=1+\alpha_{1} i^{-1 / 3}+\alpha_{2} i^{-2 / 3},
$$

where $\alpha_{1}$ and $\alpha_{2}$ are given by

$$
\begin{aligned}
& \alpha_{1}=\left[\left(\kappa_{2}-1\right)-\left(\kappa_{1}-1\right) 2^{-2 / 3}\right] /\left[2^{-1 / 3}-2^{-2 / 3}\right], \\
& \alpha_{2}=\left[\left(\kappa_{2}-1\right)-\left(\kappa_{1}-1\right) 2^{-1 / 3}\right] /\left[2^{-1 / 3}-2^{-2 / 3}\right],
\end{aligned}
$$

so that $\kappa_{i}$ can be computed for all $i$ once $\kappa_{1}$ and $\kappa_{2}$ are known. By forcing the identity of Eq. (4) with a Taylor series expansion of $\rho$ (in powers of $p$ ) and using the virial equation of state to provide the first two derivatives of density with respect to pressure, DM are able to evaluate $\kappa_{1}$ and $\kappa_{2}$ which are given by

$$
\begin{aligned}
& \kappa_{1}=-(1 / \theta) \ln \left(P_{e} / q_{0} k_{B} T\right), \\
& \kappa_{2}=-\left(1 / \theta 2^{2 / 3}\right) \ln \left[-\left(P_{e} / q_{0} k_{B} T\right)^{2} 2^{\tau-1} q_{0} B\right],
\end{aligned}
$$

where $B$ is the second virial coefficient. $\tau$ and $q_{0}$ are determined by the fluid critical properties through the relations

$$
\begin{aligned}
& p_{c}=q_{0} \zeta(\tau-1), \\
& p_{c}=q_{0} k_{B} T_{c} \zeta(\tau),
\end{aligned}
$$

where $\rho_{c}, p_{c}$, and $T_{c}$ and the critical number density, pressure, and temperature, respectively, and $\xi(x)$ is the Riemann zeta function given by

$$
\zeta(x)=\sum_{i=1}^{\infty} i^{-x}
$$

With these quantities determined, the rate of nucleation can be expressed as

$$
\begin{aligned}
J= & (1 / 3)\left\{(\theta / \pi)\left[1+\alpha_{1} i^{*-1 / 3}+9 \tau i^{*-2 / 3} / 2 \theta\right]\right\}^{1 / 2} \\
& \times p s_{1} \rho_{i *} /\left(2 \pi m k_{B} T\right)^{1 / 2},
\end{aligned}
$$

where $p$ is the partial pressure of the vapor, $m$ is the molecular mass, and $\rho_{i^{*}}$ is given by

$$
\rho_{i^{*}}=q_{0} \exp \left(-\kappa_{i *} \theta i^{* 2 / 3}-\tau \ln i^{*}+i^{*} \ln S\right) \text {. }
$$

The critical cluster size $l^{*}$ is given by the real root of the cubic equation

$$
\tau x^{3}+\alpha_{1} \theta x^{2} / 3+2 \theta x / 3-\ln S=0, \quad x=i^{*-1 / 3} .
$$

The DM expression contains the CNT as a special case and is obtained by setting $\tau=0, q_{0}=p / k_{B} T$, and $\kappa_{i}=1$.

For the acetonitrile and nitromethane calculations the virial coefficient data were taken from the collection of
TABLE II. The DM parameters $\kappa_{1}, \kappa_{2}, \kappa_{i} *$, and the critical cluster size $i^{*}$ for $\mathrm{CH}_{3} \mathrm{CN}$ and $\mathrm{CH}_{3} \mathrm{NO}_{2}$.

\begin{tabular}{rcccc}
\hline \hline$T(\mathrm{~K})$ & $\kappa_{1}$ & $\kappa_{2}$ & $\kappa_{i^{*}}$ & $i^{*}$ \\
\hline $\mathrm{CH}_{3} \mathrm{CN}$ & & & & \\
230.0 & 0.836 & 0.775 & 0.865 & 97 \\
240.0 & 0.823 & 0.768 & 0.869 & 107 \\
250.0 & 0.811 & 0.760 & 0.873 & 119 \\
260.0 & 0.799 & 0.753 & 0.876 & 131 \\
270.0 & 0.786 & 0.745 & 0.879 & 145 \\
280.0 & 0.773 & 0.737 & 0.882 & 160 \\
290.0 & 0.760 & 0.728 & 0.884 & 177 \\
300.0 & 0.747 & 0.718 & 0.886 & 196 \\
& & & & \\
$\mathrm{CH}_{3} \mathrm{NO}_{2}$ & & & & 73 \\
250.0 & 0.735 & 0.755 & 0.899 & 81 \\
260.0 & 0.730 & 0.753 & 0.902 & 90 \\
270.0 & 0.724 & 0.750 & 0.906 & 100 \\
280.0 & 0.718 & 0.747 & 0.909 & 111 \\
290.0 & 0.712 & 0.743 & 0.911 & 123 \\
300.0 & 0.706 & 0.739 & 0.914 & \\
\hline \hline
\end{tabular}

Dymond and Smith ${ }^{11}$ as included in Table I. The $B$ vs $T$ data were fit to the following functional form as suggested by DM (Ref. 9):

$$
B p_{c} / R T_{c}=(9 / 128)\left(1-6 / T_{r}^{2}\right)-a T_{r} \exp \left(b / T_{r}\right),
$$

where $a$ and $b$ are determined by least-squares fits.

The first step in the calculations is to evaluate the parameters $\tau$ and $q_{0} . \tau$ is determined from the critical compressibility since from Eqs. 8(a) and 8(b), it follows that

$$
Z_{c}^{-1}=\zeta(\tau-1) / \zeta(\tau)
$$

Values of the Riemann zeta function $\xi(x)$ for $x<2$ were taken from the interpolation of the values obtained from the DM work ${ }^{9}$ and from the published tables of the function. ${ }^{12}$ Table II includes the values of $\kappa_{1}, \kappa_{2}, \kappa_{i}^{*}$, and $i^{*}$ calculated at different temperatures for acetonitrile and nitromethane. The computer program used for the calculations was checked by reproducing the DM results for the alcohols, water, and nonane. ${ }^{9}$ Table III compares the model parameters used for acetonitrile and nitromethane with those for the substances investigated by DM. The critical supersaturations predicted by DM model for acetonitrile and nitromethane are shown in Figs. 3 and 4, respectively. Inspection of Figs. 3 and 4 clearly indicates that the DM calculations predict critical supersaturations below those of the CNT and far below the experimental results. One possible problem in these calculations is the extrapolation of the virial coefficient to temperatures as much as $70 \mathrm{~K}$ below the lowest value reported in the literature. Therefore, the effect of the uncertainty introduced by this extrapolation on the DM calculations has been investigated. It was found that a decrease in $B$ (in magnitude) by $73 \%$ from its extrapolated value at $270 \mathrm{~K}$ was required in order to increase the predicted supersaturation to the value given by the $\mathrm{CNT}$ for $\mathrm{CH}_{3} \mathrm{CN}$ at this temperature. Yet this decreased value of $-4050 \mathrm{~cm}^{3} / \mathrm{mol}$ is smaller than the literature value of -5350 at $308.2 \mathrm{~K}$, whereas it should be greater in magnitude. Therefore the 
TABLE III. Comparison of DM parameters for different substances.

\begin{tabular}{|c|c|c|c|c|c|c|c|c|c|c|}
\hline \multirow[b]{2}{*}{ Compound } & \multirow[b]{2}{*}{$Z_{c}$} & \multirow[b]{2}{*}{$\tau$} & \multirow[b]{2}{*}{$q_{0} / \rho_{c}$} & & \multicolumn{6}{|c|}{$T=270 \mathrm{~K}$} \\
\hline & & & & & $\kappa_{1}$ & $\kappa_{2}$ & $\kappa_{i *}$ & $i^{*}$ & $S_{\mathrm{DM}}$ & $S_{\mathrm{CNT}}$ \\
\hline Acetonitrile & 0.184 & 2.127 & 0.119 & & 0.786 & 0.745 & 0.879 & 145 & 2.62 & 3.07 \\
\hline Nitromethane & 0.208 & 2.148 & 0.136 & & 0.724 & 0.750 & 0.906 & 90. & 4.51 & 5.46 \\
\hline Methanol & 0.224 & 2.162 & 0.148 & & 1.31 & 1.28 & 1.09 & 151 & 2.00 & 1.79 \\
\hline Ethanol & 0.240 & 2.175 & 0.159 & & 1.14 & 1.12 & 1.04 & 121 & 2.49 & 2.30 \\
\hline$n$-propanol & 0.253 & 2.188 & 0.169 & & 1.05 & 1.04 & 1.01 & 92 & 3.43 & 3.26 \\
\hline$n$-butanol & 0.259 & 2.193 & 0.173 & & 0.997 & 0.968 & 0.967 & 80 & 4.39 & 4.64 \\
\hline Water & 0.229 & 2.166 & 0.151 & & 0.992 & 1.03 & 1.04 & 73 & 4.87 & 4.42 \\
\hline$n$-nonane & 0.256 & 2.190 & 0.171 & - & 0.639 & 0.716 & 0.908 & 56 & 11.0 & 15.3 \\
\hline Acetonitrile $^{\mathrm{a}}$ & & & & & 1.47 & 1.44 & 1.19 & 67 & 4.46 & 3.07 \\
\hline Nitromethane $\mathrm{a}^{\mathrm{a}}$ & & & & & 1.21 & 1.19 & 1.08 & 56 & 6.99 & 5.46 \\
\hline
\end{tabular}

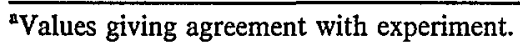

error introduced by the extrapolation cannot account for the significant differences in the predictions of the DM model and the CNT or the differences between the DM model and the experimental results. It was also found that the DM model is not especially sensitive to the values of $\tau$ and $q_{0}$ and Table IV shows that changing these parameters by $\sim 25 \%$ has a negligible effect on the calculated supersaturations $(\sim 2 \%-3 \%)$.

The most likely reason for the higher nucleation rates predicted by the DM model for acetonitrile and nitromethane lies in the evaluation of the parameters $\kappa_{1}$ and $\kappa_{2}$. From their studies DM find that $\kappa_{i}$ characteristically converges to unity from values greater than unity for polar substances and from less than unity for nonpolar substances, in qualitative agreement with the calculations of
Sinanoglu. ${ }^{13}$ Unfortunately, $\kappa_{1}$ and $\kappa_{2}$ as calculated for acetonitrile and nitromethane via Eq. (7) are less than unity for the temperature ranges of the nucleation experiments and this leads to predictions for the critical supersaturations well below those of the CNT. The same effect is found in the DM calculations for the critical supersaturations of nonane. Of all the substances investigated in Ref. 9, nonane is the only substance with $\kappa_{i} *$ less than unity throughout the temperature range of the calculations. Subsequently, the DM critical supersaturations for nonane are below those of the CNT in contrast to other substances where $\kappa_{i}$ * is greater than unity. Also, the DM predictions for the critical supersaturations of $n$-butanol are lower than the predictions of the CNT only at lower temperatures where $\kappa_{i} *$ is less than unity.

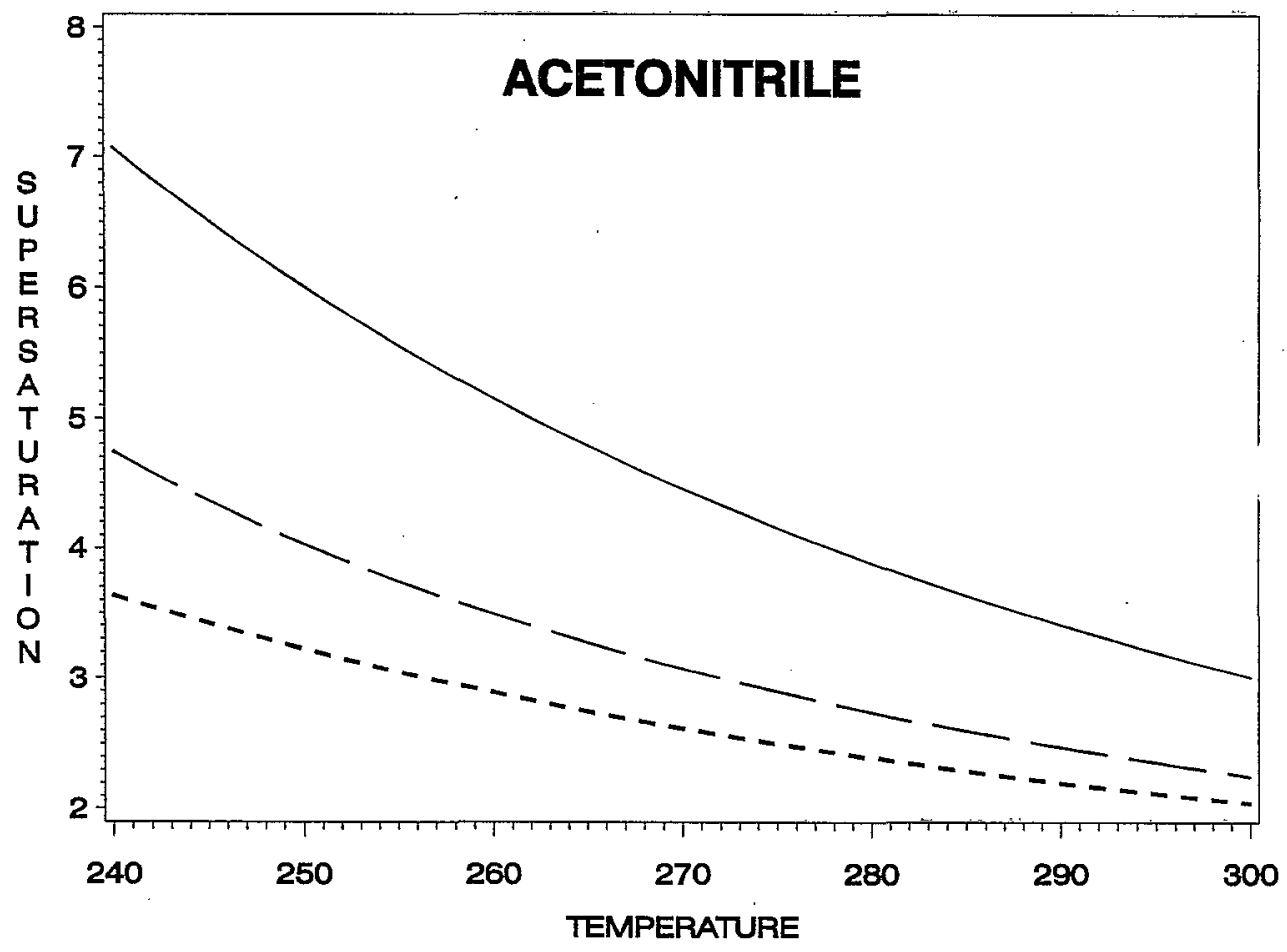

FIG. 3. Critical supersaturation vs temperature for acetonitrile. Solid line represents the experimental results, dashed line the CNT, and the dotted line the predictions of the DM model. 


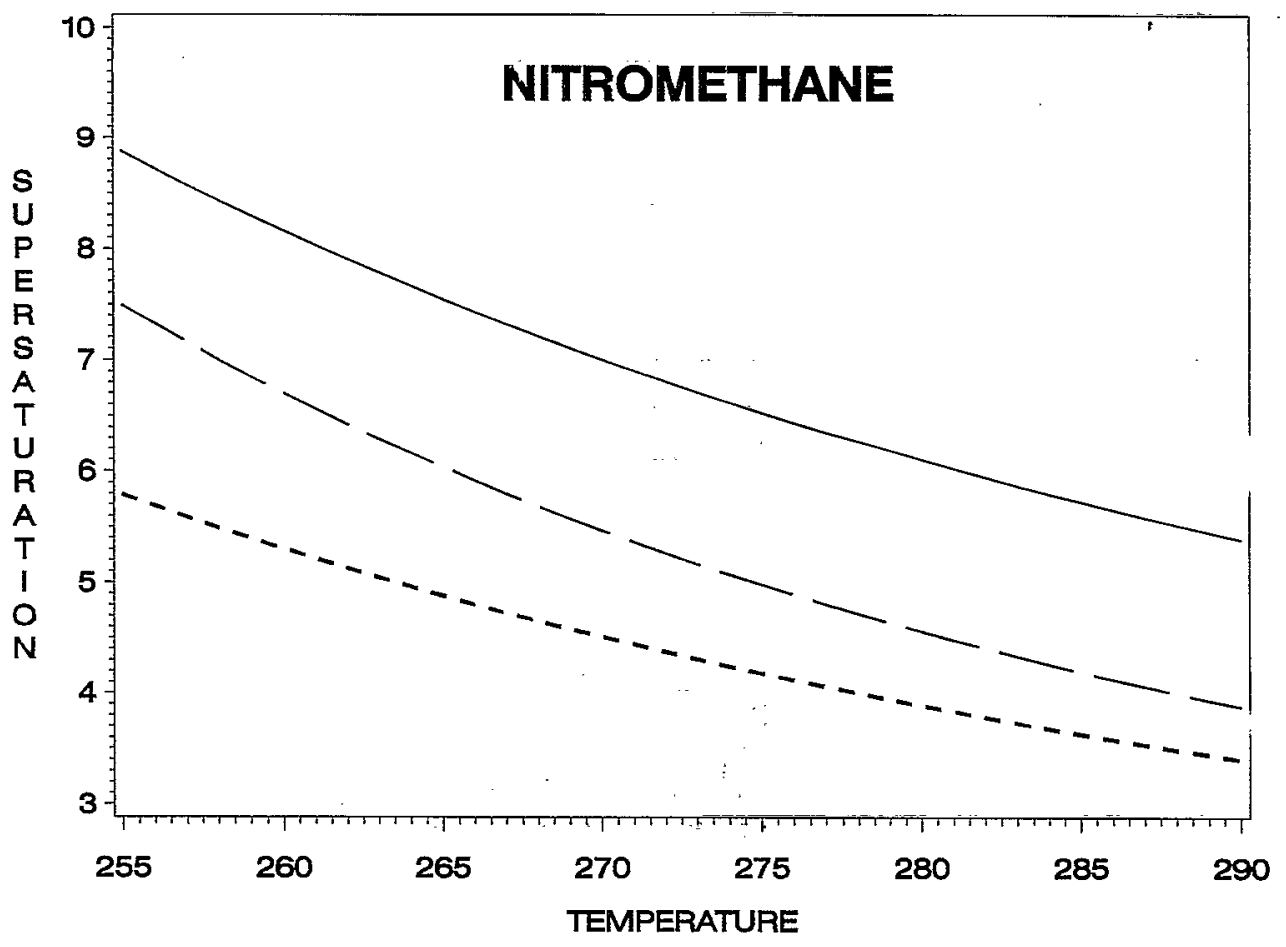

FIG. 4. Critical supersaturation vs temperature for nitromethane. Solid line represents the experimental results, dashed line the CNT, and the dotted line the predictions of the DM model.

It is interesting to note that our experimental data in conjunction with the DM model would suggest $\kappa_{1}$ values (at $T=270 \mathrm{~K}$ ) of 1.47 and 1.21 for acetonitrile and nitromethane, respectively, as shown in Table III (other similar values are possible depending upon the choice of $\kappa_{2}$ ). These values converge to a value close to unity (as they should) at the critical size. The predicted values also indicate that $\kappa_{i}$ decreases with decreasing dipole moment in agreement with the calculations of Sinanoglu for several classes of substances. ${ }^{13} \mathrm{It}$ is clear that more reliable ways to

TABLE IV. Dependence of the calculated supersaturations on $\tau$ and $q_{0} / \rho_{c}$ for acetonitrile and nitromethane.

\begin{tabular}{lllll}
\hline \hline$\tau$ & $q_{0} / \rho_{c}$ & $T(\mathrm{~K})$ & $S_{\mathrm{DM}}$ & $\%$ change in $S_{\mathrm{DM}}$ \\
\hline $\mathrm{CH}_{3} \mathrm{CN}$ & & & & \\
& & & & \\
& & 250.0 & 3.14 & -2.5 \\
& & 270.0 & 2.56 & -1.9 \\
& & 290.0 & 2.16 & -1.8 \\
& & 250.0 & 3.30 & +2.5 \\
& & 290.0 & 2.25 & +2.7 \\
$\mathrm{CH}_{3} \mathrm{NO}_{2}$ & & & & \\
$2.69(+25 \%)^{\mathrm{a}}$ & 0.143 & 250.0 & 6.16 & -3.0 \\
& & 270.0 & 4.38 & -2.9 \\
& & 290.0 & 3.31 & -2.6 \\
$1.61(-25 \%)^{\mathrm{a}}$ & 0.0910 & 250.0 & 6.55 & +3.1 \\
& & 270.0 & 4.64 & +2.9 \\
& & 290.0 & 3.49 & +2.6 \\
\hline \hline
\end{tabular}

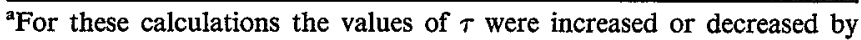
$25 \%$ and $q_{0} / \rho_{c}$ was recalculated using the adjusted $\tau$ values. calculate the surface energy correction factor $\kappa_{i}$ are needed before a final conclusion can be reached regarding the application of the DM model to highly polar substances.

\section{THE ORIENTED-DIPOLES MODEL OF ABRAHAM}

In the oriented-dipole model (due to $A b r a h a m{ }^{10}$ ), one explicitly includes the effect of dipole-dipole interaction on the surface free energy of the embryonic droplets. It assumes that for highly polar liquids there is preferential orientation of the surface molecules. Frenkel ${ }^{14}$ has suggested that surface molecules will tend to orient themselves so that their electric fields are within the high-dielectric constant liquid in order to minimize the field free energy. Thus for a planar surface the surface dipoles are taken as exhibiting a parallel alignment. For a sphere the size of a condensation nucleus this orientation is disturbed by the large curvature of the surface. The suggested dipole alignment at the planar and spherical interfaces is illustrated below.

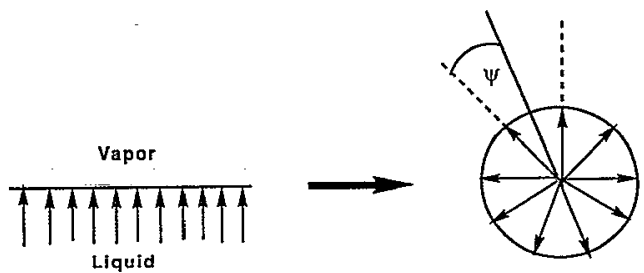

The angle $\psi$ between any two nearest-neighbor surface dipoles is directly related to the droplet size. The deviation from parallel orientation involves an increase in the surface potential energy which is given by 


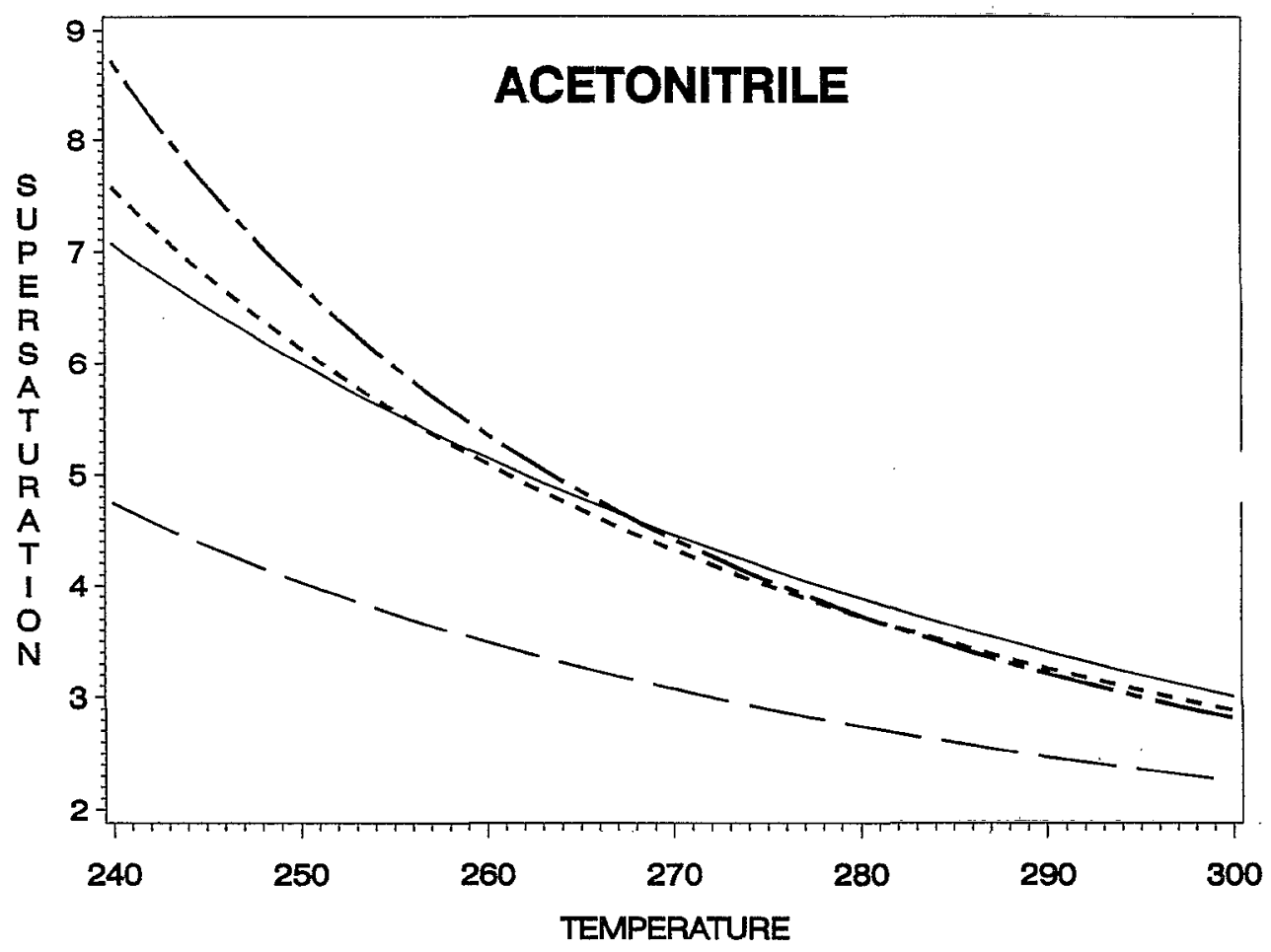

FIG. 5. Critical supersaturation vs temperature for acetonitrile. Solid line represents the experimental results, dashed line the CNT, dotted line the prolate spheroidal model $(e=0.950)$, and the dash-dot-dash line the oriented dipoles model $(d=3.7, c=2)$.

$$
U_{s}=\left(\mu^{2} / 2\right)(1-\cos \psi) c j_{s} / 2 d^{3},
$$

where $\mu$ is the magnitude of the dipole moment, $c$ is the number of surface nearest neighbors of a surface molecule, $d$ is the distance between nearest neighbors, and $j_{s}$ is the total number of molecules at the surface of the droplet. Abraham showed that for small angles $\psi\left(1-\cos \psi \approx \psi^{2}\right.$ / 2), Eq. (14) can be approximated as

$$
U_{s}=\left(\mu^{2} c / 2 d^{3}\right)[(1-\cos \psi) /(1-\cos \psi / 2)] \approx 2 \mu^{2} c / d^{3} .
$$

Equation (15) represents a constant correction to the surface free energy of the clusters due to dipole-dipole interaction within a curved surface. Therefore, the total surface free energy, $F_{n}^{(s)}$, of a cluster of surface area $A$ and radius $r$ is now given by

$$
F_{n}^{(s)}=\sigma(r) A=\sigma_{0} A+U_{s},
$$

where $\sigma(r)$ is a size dependent surface tension due to the effect of surface curvature and $\sigma_{0}$ is the bulk planar surface tension assumed by the CNT. This size dependent surface tension can be expressed as

$$
\sigma(r)=\sigma_{0}+2 \mu^{2} c /\left(4 \pi r^{2} d^{3}\right),
$$

where $r$ is the cluster radius.

Using the literature values of the dipole moment (shown in Table VI of the previous paper), assuming the number of surface nearest neighbors $c=2$ and the distance between nearest neighbors $d=3.7-4.5 \AA$, we obtained the size dependent surface tension for the clusters from Eq. (17). As $r$ in Eq. (17) must be calculated from $S_{\mathfrak{c}}$, itera- tions are performed until $\sigma(r), r$, and $S_{c}$ all are consistent with the Kelvin relation. Figures 5-8 show the predictions of the CNT when this surface tension $\sigma(r)$ is used in place of $\sigma_{0}$. Similar curves can also be generated with $c=3$ and slightly larger values of $d$. As can be seen from the figures, appropriate choices of $c$ and $d$ can yield fair agreement with experiment, although the temperature dependence is somewhat too sharp at lower temperatures. It is possible that the smaller critical cluster sizes that accompany lower temperatures may not be large enough to favor the suggested orientation. Also, as the droplet size decreases the $\cos \psi$ approximation becomes less valid.

\section{NONSPHERICAL CLUSTERS}

Another attempt to rationalize the experimental results is to assume that for highly polar molecules the clusters may become somewhat nonspherical to allow for greater end-to-end and antiparallel alignment of the dipoles (including those in the interior of the drop). Elongation of the clusters would tend to give them a prolate spheroidal shape. With this possibility in mind we have generalized the CNT to allow clusters of this shape. What this amounts to is the substitution of the expressions for the surface area and volume of a prolate spheroid for those of the sphere assumed by the CNT, everywhere the spherical assumption is invoked. Following the CNT we can express the Gibbs free energy of formation of the droplet as

$$
\Delta G=\sigma A-V \rho_{1} k_{B} T \ln S,
$$




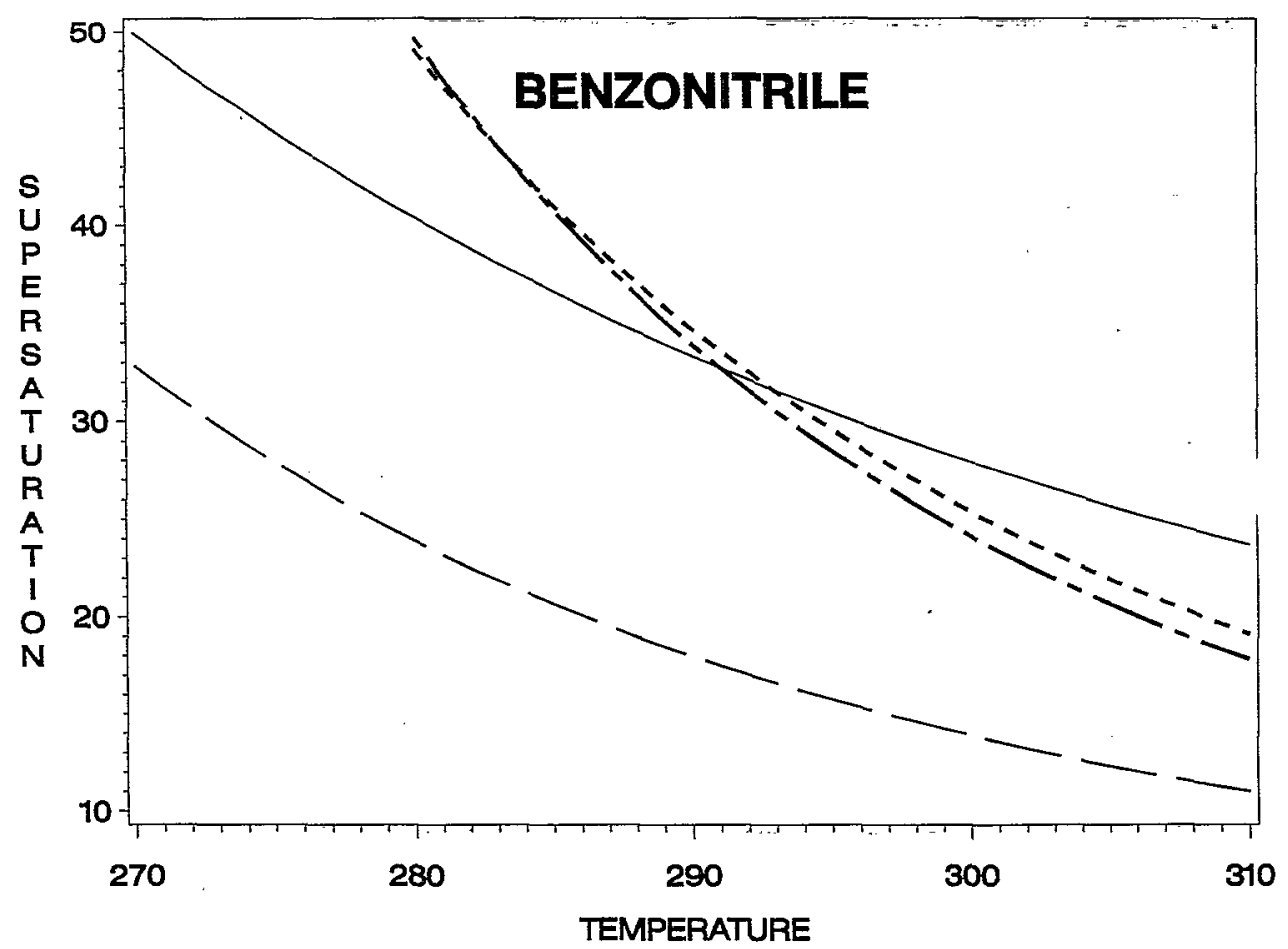

FIG. 6. Critical supersaturation vs temperature for benzonitrile. Solid line represents the experimental results, dashed line the CNT, dotted line the prolate spheroidal model $(e=0.944)$, and the dash-dot-dash line the oriented dipoles model $(d=4.4, c=2)$.

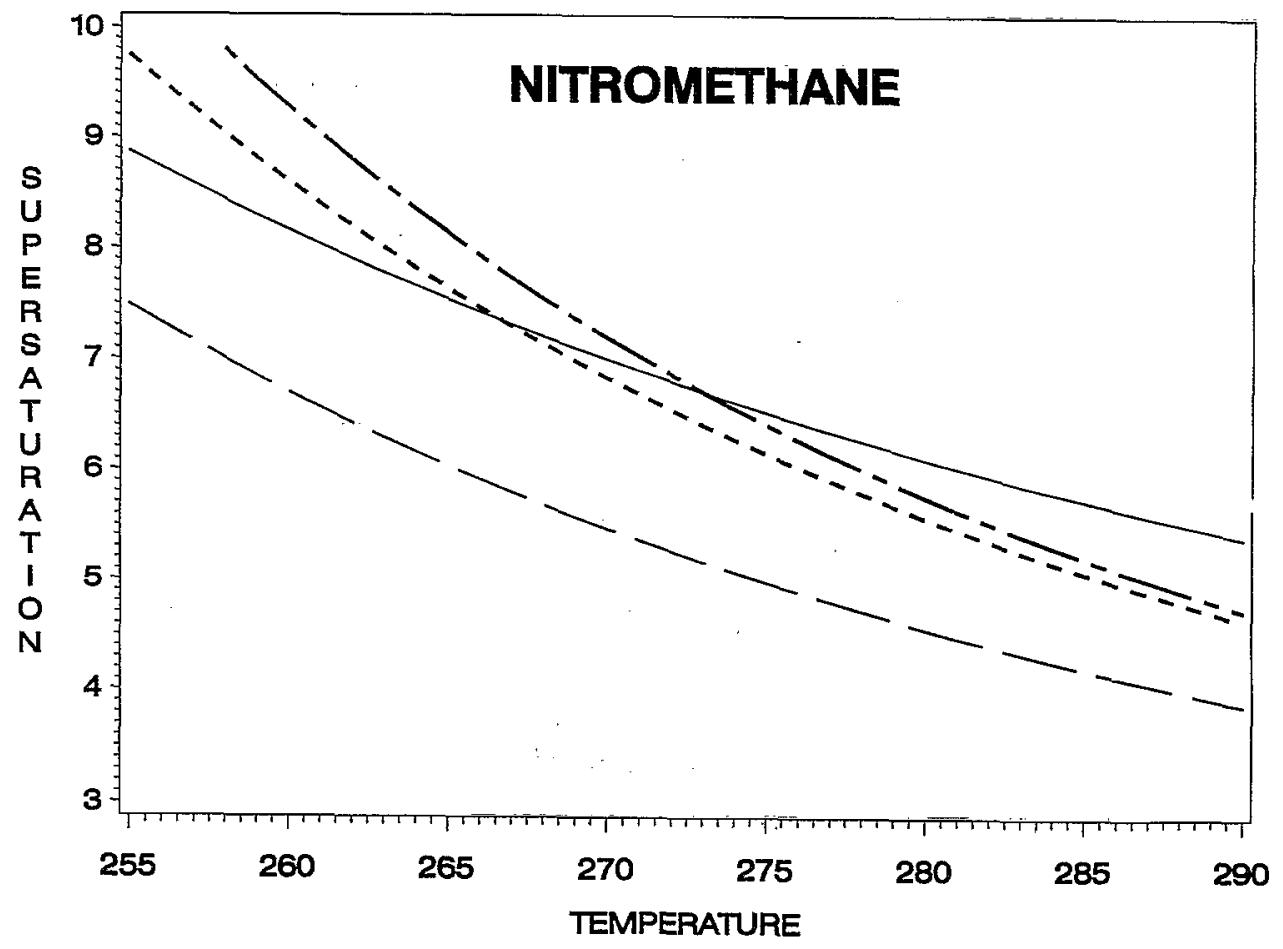

FIG. 7. Critical supersaturation vs temperature for nitromethane. Solid line represents the experimental results, dashed line the CNT, dotted line the prolate spheroidal model $(e=0.883)$, and the dash-dot-dash line the oriented dipoles model $(d=4.2, c=2)$. 


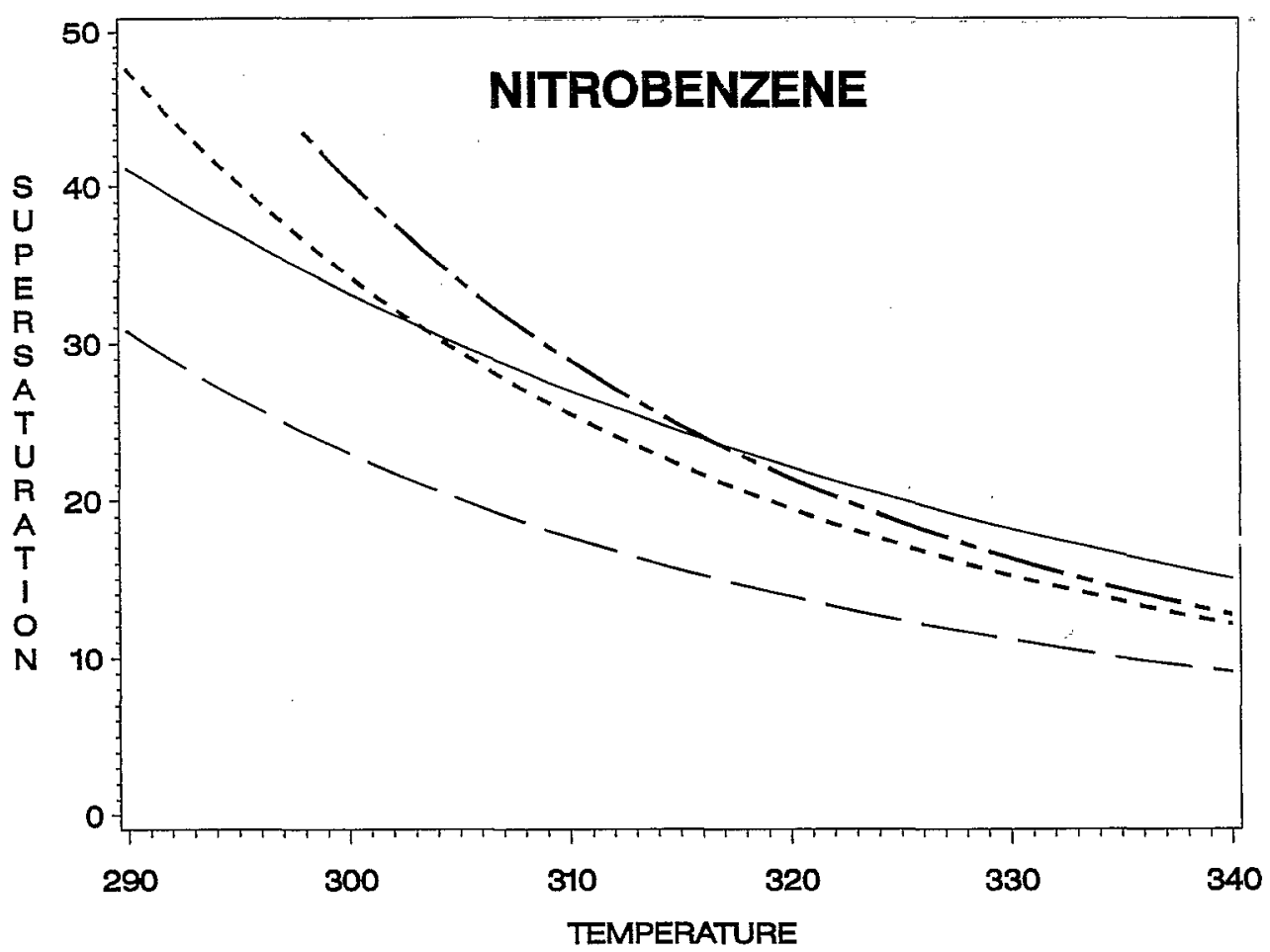

FIG. 8. Critical supersaturation vs temperature for nitrobenzene. Solid line represents the experimental results, dashed line the CNT, dotted line the prolate spheroidal model $(e=0.883)$, and the dash-dot-dash line the oriented dipoles model $(d=4.5, c=2)$.

where $V$ is the volume of the droplet and $\rho_{1}$ is the liquid number density. For a prolate spheroid $A$ and $V$ are given by $^{15}$

$$
\begin{aligned}
& A=2 \pi b^{2}+(2 \pi a b / e) \sin ^{-1} e, \\
& V=4 \pi a b^{2} / 3,
\end{aligned}
$$

where $a$ and $b$ are the semimajor and semiminor radii, respectively, and $e$ is the eccentricity of the ellipse used to generate the prolate spheroid (by rotation about its major axis). Defining

$$
\delta=b / a=\left(1-e^{2}\right)^{1 / 2}
$$

and

$$
\alpha=(2 \pi / e) \sin ^{-1} e,
$$

we can write

$$
\Delta G(a)=4 \pi \xi a^{2} \sigma-(4 \pi / 3) \delta^{2} a^{3} \rho_{1} k_{B} T \ln S,
$$

where

$$
\xi=\delta^{2}(1+\alpha / 2 \pi \delta) / 2,
$$

and $a$ is the variable that indicates cluster size. In the usual manner, the barrier height to nucleation is determined by the condition

$$
\left.(\partial \Delta G / \partial a)\right|_{a=a *}=0
$$

which gives

$$
a^{*}=2 \sigma \xi /\left(\delta^{2} \rho_{1} k_{B} T \ln S\right),
$$

where $a^{*}$ is the semimajor radius of the critical sized cluster. Using Eq. (26) in Eq. (23) gives the barrier height as

$$
\Delta G_{\mathrm{ps}}^{*}=16 \pi \lambda \sigma^{3} /\left[3\left(\rho_{1} k_{B} T \ln S\right)^{2}\right]=\lambda \Delta G_{s}^{*},
$$

where $\lambda$ is given by

$$
\lambda=\xi^{3} / \delta^{4}=\left[\delta+\left(\sin ^{-1} e\right) / e\right]^{3} / 8 \delta
$$

and the subscripts ps and $s$ refer to the prolate spheroidal and spherical droplet models, respectively. It is easily shown that in the limit that $e \rightarrow 0$ (prolate spheroid $\rightarrow$ sphere) $\lambda=1$ and the classical expression for the barrier height associated with spherical droplets is completely recovered.

The rate of nucleation can be expressed in the form

$$
J=C_{i *} Z_{i *} \rho_{v} \exp \left[-\Delta G^{*} / k_{B} T\right],
$$

where $C_{i^{*}}$ is the condensation rate, $Z_{i^{*}}$ is the so-called "Zeldovich factor" and $\rho_{v}$ is the vapor number density. For prolate spheroidal clusters one obtains

$$
C_{i *_{\mathrm{ps}}}=4 \pi a^{* 2} \xi q p /\left(2 \pi m k_{B} T\right)^{1 / 2}=\lambda C_{i *_{s}}
$$

and

$$
Z_{i * \mathrm{ps}}=\lambda^{1 / 6}\left\{4 \sigma\left(3 / 4 \pi \rho_{1}\right)^{2 / 3} /\left[9 k_{B} T i^{* 4 / 3}\right]\right\}^{1 / 2}=\lambda^{1 / 6} Z_{i *_{s}}
$$

which yields for the rate equation 


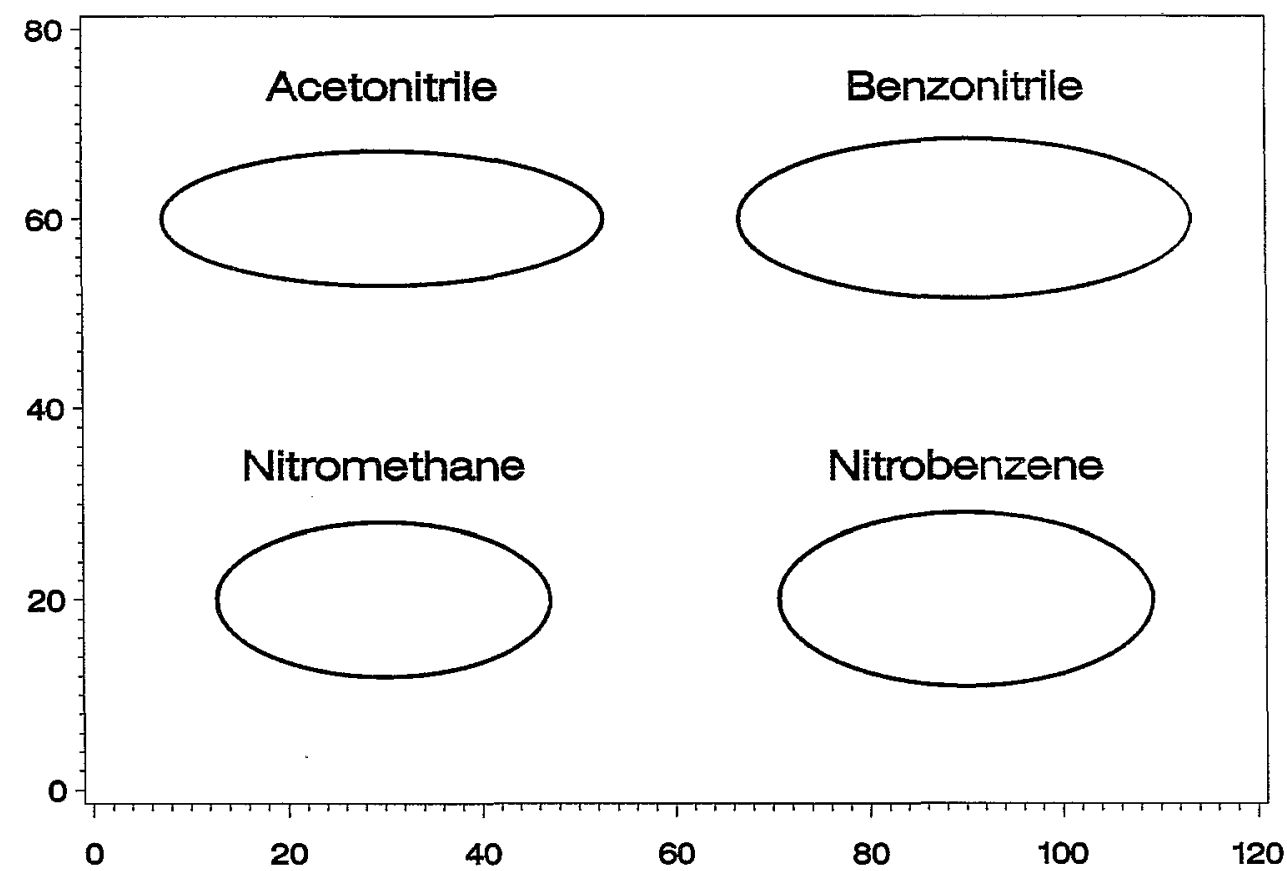

FIG. 9. Critical cluster shapes at $T_{r}=0.44$ for the polar compounds. Scale in $\AA$. Eccentricities are acetonitrile $(0.950)$, benzonitrile $(0.944)$, nitromethane $(0.883)$, and nitrobenzene $(0.883)$.

$$
\begin{aligned}
J_{\mathrm{ps}}= & \lambda^{7 / 6} C_{i *_{s}} Z_{i *_{s}} \rho_{v} \exp \left[-\lambda \Delta G_{s}^{*} / k_{B} T\right] \\
= & \lambda^{7 / 6}(q / d)\left(2 N_{A}^{3} \sigma M / \pi\right)^{1 / 2}\left(S P_{e} / R T\right)^{2} \\
& \times \exp \left\{-16 \pi \lambda N_{A} M^{2} \sigma^{3} /\left[3(d \ln S)^{2}(R T)^{3}\right]\right\},
\end{aligned}
$$

where all the quantities were defined in the paragraph following Eq. (1). For the calculations the eccentricity is entered as a parameter and is a constant for a given calculation. Figure 9 shows the eccentricities and resulting cluster shapes required to generate the curves shown in Figs. $5-8$. The values of $e$ were selected to give reasonable agree- ment with experiment and it is interesting to note that both $\mathrm{CH}_{3} \mathrm{CN}$ and $\mathrm{C}_{6} \mathrm{H}_{5} \mathrm{CN}$ require $e \approx 0.95$ and $\mathrm{CH}_{3} \mathrm{NO}_{2}$ and $\mathrm{C}_{6} \mathrm{H}_{5} \mathrm{NO}_{2}$ both require $e \approx 0.88$.

As can be noted from Fig. 9, a rather large degree of elongation is required to give fair agreement with the experimental results, especially for the nitriles. Figure 10 shows a hypothetical (low $T$ ) $\mathrm{CH}_{3} \mathrm{CN}$ cluster structure where the predominant dimer geometries are head-to-tail and antiparallel structures. Note that this cluster structure is quite similar to that of the completely ordered crystal where each head-to-tail chain is surrounded by four chains running antiparallel to it. ${ }^{16}$ Bohm et al. ${ }^{17}$ have performed

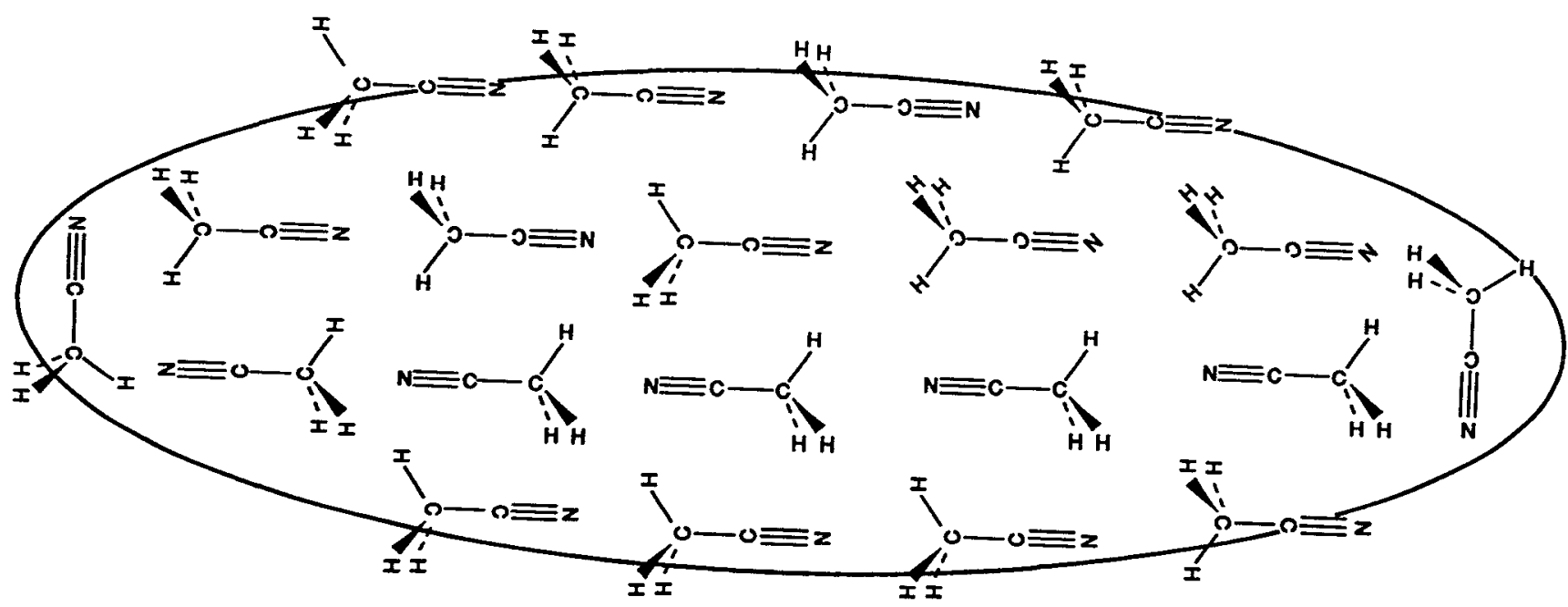

FIG. 10. Hypothetical acetonitrile cluster at low temperature showing energetically favorable head-to-tail, antiparallel, and displaced parallel alignments for both the surface and the interior molecules of the droplet. 
molecular dynamics simulations of bulk liquid acetonitrile at temperatures of 291 and $258 \mathrm{~K}$ and for their potential model the antiparallel, head-to-tail, T-shaped, and parallel dimer structures have potential well depths of about -21 , $-12,-5$, and $-4 \mathrm{~kJ} / \mathrm{mol}$, respectively. Clearly, the antiparallel and head-to-tail alignments are the most favorable structures. Jorgensen et $a l .^{18}$ have performed Monte Carlo simulations of $\mathrm{CH}_{3} \mathrm{CN}$ and have further investigated the liquid's structure. Performing simulations at 298 and 343 $\mathrm{K}$ with both the full potential and with a potential having the partial charges set to zero ( $298 \mathrm{~K}$ only), they find a sharp increase in the first peak in the $\mathrm{N}-\mathrm{CH}_{3}$ radial distribution function upon "turning on" the charges, indicating the importance of the electrostatic interactions in determining the liquid's structure. Examining a stereoplot of an arbitrary equilibrated configuration, Jorgensen notes that a particular (but typical) $\mathrm{CH}_{3} \mathrm{CN}$ molecule has two antiparallel neighbors above it, a parallel neighbor below it and to the left (displaced parallel), and it can be considered as part of a distorted chain spanning at least the length of the simulation box $(\approx 20 \AA)$. Jorgensen thus concludes that remnants of the short range order in the crystal are apparent in the liquid at $298 \mathrm{~K}$, and we would expect that ordering to persist more strongly at the temperatures (240$290 \mathrm{~K}$ ) of the nucleation experiments. In fact, at temperatures near the freezing point $(229 \mathrm{~K})$ direct formation of microcrystals from the vapor can be observed. Jorgensen also notes, however, that order beyond the nearest neighbor level is weak at $298 \mathrm{~K}$ except for some winding chains and stacked multimers.

All of this discussion, of course, takes no account of surface effects on the cluster structure. Shreve et al. ${ }^{19}$ have conducted a molecular dynamics study of liquid drops composed of Stockmayer molecules and although they do not find any evidence of a preferred surface orientation in droplets composed of 50 molecules, their simulations of systems composed of 260 and 896 molecules indicate that the dipoles tend to lie parallel to the droplet surface, which would be consistent with the orientation suggested for the elongated cluster shown in Fig. 10. In comparing the properties of $\mathrm{CH}_{3} \mathrm{CN}$ clusters with those composed of Stockmayer molecules it is worth noting that Stockmayer particles possess a single Lennard-Jones center to which is added a term giving the potential a directional character and it seems unlikely that comparative cluster sizes would correspond to the same number of molecules in the clusters. Thus the typical critical cluster sizes of 50-150 molecules for $\mathrm{CH}_{3} \mathrm{CN}$ may be large enough to favor a dipole orientation parallel to the cluster surface.

\section{SUMMARY AND CONCLUSIONS}

In the present work we have examined several models to explain the strong deviations observed in the homogeneous nucleation of highly polar liquids from the predictions of the classical nucleation theory. The observed increase in the critical supersaturations cannot be explained in terms of vapor association within the scope of the KSR model since unrealistically higher association constants would be required in order to bring an agreement between experiment and theory. The failure of the refined droplet model of Dillmann and Meier is attributed to incorrect evaluation of the size dependent surface energy correction factor $\kappa_{i}$. Reliable ways to calculate this parameter for highly polar substances are necessary in order to critically evaluate the generality of the DM model.

The dipole orientation and elongated cluster models seem to give reasonable (but not complete) representation of the experimental results. This suggests that in the homogeneous nucleation of highly polar substances at lower temperatures, dipole orientation may result in increased surface tension or in elongation of the spherical droplets, thus increasing the energy barrier and the critical supersaturation relative to weakly or nonpolar systems. This appealing picture needs to be thoroughly examined by molecular simulations of highly polar clusters in a nucleating system. We are now actively pursuing this direction in order to establish a detailed understanding of the homogeneous nucleation of highly polar substances.

\section{ACKNOWLEDGMENTS}

Acknowledgment is made to the donors of the Petroleum Research Fund, administered by the American Chemical Society and to the Thomas F. and Kate Miller Jeffress Memorial Trust and to Philip Morris USA for the partial support of this research. We thank Dr. Cliff Lilly and Dr. Peter Lipowicz (Philip Morris Research Center, Richmond, VA) for their continuous encouragement.

${ }^{1}$ D. Wright, R. Caldwell, and M. S. El-Shall, Chem. Phys. Lett. 176, 46 (1991).

${ }^{2}$ D. Wright and M. S. El-Shall, Chem. Phys. Lett. 189, 103 (1992).

${ }^{3}$ D. Wright, R. Caldwell, and M. S. El-Shall, J. Chem. Phys. 98, 3356 (1993).

${ }^{4}$ R. H. Heist, K. M. Colling, and C. S. DuPois, J. Chem. Phys. 65, 5147 (1976).

${ }^{5}$ Y. G. Russell and R. H. Heist, J. Chem. Phys. 69, 3723 (1978).

${ }^{6}$ G. Agarwal and R. H. Heist, J. Chem. Phys. 73, 902 (1980).

${ }^{7}$ J. L. Katz, H. Saltzburg, and H. Reiss, J. Colliod Interface Sci. 21, 560 (1966).

${ }^{8}$ A. Dillmann and G. E. A. Meier, Chem. Phys. Lett. 160, 71 (1989).

${ }^{9}$ A. Dillmann and G. E. A. Meier, J. Chem. Phys. 94, 3872 (1991).

${ }^{10}$ F. F. Abraham, Homogeneous Nucleation (Academic, New York, 1974); J. Chem. Phys. 50, 3977 (1969); Science 168, 833 (1970).

$11 \mathrm{~J}$. H. Dymond and E. B. Smith, The Virial Coefficients of Pure Gases and Mixtures (Clarendon, Oxford, 1980); For $\mathrm{CH}_{3} \mathrm{CN}$ data sets 2, 3 $(313.15-353.25 \mathrm{~K})$, and 4 were used; For $\mathrm{CH}_{3} \mathrm{NO}_{2}$ data sets 1,2 , and 3 were used.

${ }^{12}$ Jahne-Emde-Losch, Tables of Higher Functions (McGraw-Hill, New York, 1960).

${ }^{13}$ O. Sinanoglu, J. Chem. Phys. 75, 463 (1981).

${ }^{14} \mathrm{~J}$. Frenkel, Kinetic Theory of Liquids (Dover, New York, 1946).

${ }^{15}$ CRC Handbook of Mathematical Sciences, 5th ed., edited by W. H. Beyer (Chemical Rubber, Boca Raton, 1980).

${ }^{16}$ H. Michel and E. Lippert, Organic Liquids: Structure, Dynamics, and Chemical Properties, edited by A. D. Buckingham, E. Lippert, and S. Bratos (Wiley, New York, 1978), Chap. 17.

${ }^{17}$ H. J. Bohm, I. R. McDonald, and P. A. Madden, Mol. Phys. 49, 347 (1983).

${ }^{18}$ W. L. Jorgensen and J. M. Briggs, Mol. Phys. 63, 547 (1988).

${ }^{19}$ A. P. Shreve, J. P. R. B. Walton, and K. E. Gubbins, J. Chem. Phys. 85, 2178 (1986).

${ }^{20}$ L. A. Curtiss, D. J. Frurip, and M. Blander, J. Phys. Chem. 86, 1120 (1982).

${ }^{21}$ A. Ksiazczak and A. Anderko, Ber. Bunsenges. Phys. Chem. 91, 1048 (1987). 